\title{
Problemática del mejoramiento por hibridación en nopal (Opuntia spp): hipótesis sobre segregación mendeliana poliploide
}

\author{
Problematic of breeding by hybridization in cactus pear (Opuntia spp): hypothesis about \\ polyploid mendelian segregation
}

Carlos Alberto Núñez-Colín ${ }^{1 *}$, Clemente Gallegos-Vázquez², Candelario Mondragón-Jacobo ${ }^{3}$

RESUMEN

Opuntia es un género complejo y con importancia hortícola: tiene reportadas diversas complicaciones reproductivas, como poliploidía y apomixis; además, presenta problemáticas con el mejoramiento por hibridación debido a su baja efectividad, la cual se atribuye a la apomixis como método de propagación vegetativa. No obstante, la segregación típica de un poliploide podría explicar mejor este fenómeno más que otros problemas reproductivos, aun si éstos estuvieran presentes. Es por lo anterior que el presente ensayo deriva la segregación de un locus con dos y con tres alelos y designa las probabilidades de heterocigocidad en el sistema octoploide con el fin de clarificar un poco la posible segregación a obtener mediante hibridación en nopal. Por el hecho de ser poliploide, el nopal presenta alta heterocigocidad y genera, para cada locus, desde nueve genotipos diferentes (dos alelos) hasta 6,435 genotipos diferentes (ocho alelos). Dado que no se conoce si existe en características de nopal dominancia completa, codominancia, dominancia parcial o sobredominancia, es difícil predecir su herencia, como es posible en diploides. Esto tampoco es posible en cálculos para validar genes de herencia simple. Por ello, es importante generar estos modelos para fortalecer los programas de mejoramiento genético a nivel mundial.

PALABRAS CLAVE

genética de poblaciones, alelos múltiples, mejoramiento genético

\begin{abstract} generate these models to improve the breeding programs of cactus pear worldwide.

KEYWORDS

population genetics, multiple alleles, breeding of polyploids

\footnotetext{
${ }^{1}$ Programa de Ingeniería en Biotecnología, Universidad de Guanajuato. Celaya, Guanajuato, México.

${ }^{2}$ Centro Regional Universitario Centro Norte, Universidad Autónoma Chapingo. Zacatecas, Zacatecas, México.

${ }^{3}$ Facultad de Ciencias Naturales, Univer $\neg$ sidad Autónoma de Querétaro. Querétaro, México.

*Autor para correspondencia. Mutualismo 303, col. La Suiza, A. P. 61.38060 Celaya, Guanajuato, México.

Correo electrónico: lit007a@gmail.com
}

Opuntia is a complex genus with horticultural importance: it has been reported several reproductive complications as polyploidy and apomixis. In addition, cactus pear has problems with the breeding by hybridization because it has a low effectivity, which was attributed to apomixis like an asexual propagation method; nevertheless, the typical segregation of a polyploid would explain this phenomenon in better way than other reproductive complications, even these exist. That is why the present research derived the Mendelian inherence for one locus with two and three alleles and calculated the probabilities of heterozygosity in an octoploid system to clarify the possible segregation to obtain in a cactus pear hybridization. Cactus pear by the fact of to be polyploid showed a high heterozygosity and it can generate in a model of one locus from nine different genotypes (two alleles) to 6435 different genotypes (eight alleles). As it was not known if in the cactus pear traits there are in complete dominance, codominance, partial dominance, or over dominance (heterosis) then it is difficult to predict its inherence, like it is possible in diploids, and it is also complicated the calculations to validate genes of single inherence. Because of that, it is important to

Recibido: 13 de febrero de 2018

Aceptado: 14 de mayo de 2018 


\section{INTRODUCCIÓN}

Opuntia (Cactaceae) es un género complejo que incluye especies usadas por sus cladodios jóvenes llamados "nopalitos", obtenidos principalmente de O. ficus-indica (L.) Mill., o por su producción de fruta (de muchas especies) conocidas como tunas o nostli, para el caso de los frutos dulces y xoconostles para el caso de los frutos ácidos. Los xoconostles difieren de las tunas en que presentan pericarpio grueso y pulpa casi ausente y muy ácida, además de una amplia vida de anaquel. La palabra "nopal" se refiere a la mayoría de las especies de Opuntia sin importar si es usada como fruta, nopalito o forraje (Gallegos-Vázquez et al. 2011).

En la producción de tuna en México participan 17 estados, pero sólo en ocho se concentra $87 \%$ de la superficie y $96 \%$ de la producción. Estos ocho estados se agrupan en tres regiones de producción: Sur (Puebla), Centro (Estado de México e Hidalgo) y Centro-Norte (Zacatecas, San Luis Potosí, Guanajuato, Jalisco y Aguascalientes) (Gallegos-Vázquez et al. 2009).

En el nopal existen pocos esfuerzos por estudiar su herencia y plantear un programa de mejoramiento genético sustentable, teniendo pocos antecedentes serios en la materia, entre los que destacan los hechos en la Universidad Autónoma Agraria Antonio Narro en la década de los sesenta y la obtención de la serie COPENA por parte del finado Dr. Facundo Barrientos en el Colegio de Postgraduados en los setenta (Mondragón-Jacobo 2001a). Sin embargo, en la actualidad existen programas activos de mejoramiento genético de nopal en distintas partes del mundo, por ejemplo, en Brasil (nopal forrajero), Italia (nopal tunero), Estados Unidos (nopal tunero: D'Arrigo Bros., compañía privada) y Túnez (nopal forrajero) (Móndragón-Jacobo 2001a). Además de interés por iniciar el mejoramiento en Sudáfrica e India.

Sin embargo, la tuna se ha estancado en la categoría de fruta de importancia secundaria en la preferencia de los consumidores, tanto nacionales como extranjeros; esto principalmente se debe a que el cultivo de nopal en México se apoya completamente en la utilización de variedades tradicionales obtenidas por los productores y las variedades actuales están siendo rebasadas por las demandas de mejor calidad de fruta y amplitud de oferta (Gallegos-Vázquez et al. 2009).

Para plantear un programa de mejoramiento genético se debe de tener en cuenta la biología reproductiva de la especie, entre ellas el tamaño de genoma y el nivel de ploidía. En el caso del nopal, se ha reportado como octoploide en la mayoría de sus especies, aunque se mencionan en menor medida hexaploides, tetraploides $\mathrm{y}$, aunque raros, también algunos diploides (Segura et al. 2007); sin embargo, podrían también existir triploides, pentaploides $\mathrm{y}$ heptaploides, principalmente en genotipos que aunque presentan flores no presentan fruto como el caso de la variedad 'tapón macho' (O. robusta Wendl. var. robusta).

La mayoría de los métodos tradicionales están basados en diploides, incluso las nuevas metodologías como la aplicación de marcadores de ADN, y todo su análisis, especialmente de los llamados "marcadores dominantes", se basa en el modelo de un locus diploide con número bajo de alelos, normalmente dos. No obstante, cuando se está estudiando un modelo poliploide no siempre este enfoque es correcto y puede llevar a sesgos importantes en evaluar el comportamiento de las progenies híbridas. Más aún, varios autores mencionan que en el caso del nopal existen otras complicaciones reproductivas como apomixis (García-Aguilar y Pimienta-Barrios 1996; Vélez-Gutiérrez y Rodríguez-Garay 1996; Mondragón-Jacobo 2001a, 2001b); sin embargo, esta apomixis no ha sido debidamente comprobada porque la falta de heterogeneidad en una progenie híbrida de un poliploide puede deberse a la segregación mendeliana.

Es por esto, que el presente ensayo tiene como objetivo desarrollar y clarificar la segregación mendeliana de un octoploide para confrontarlo con la falta de éxito en el mejoramiento por hibridación del nopal.

\section{MODELO DE UN LOCUS CON DOS ALELOS EN UN OCTAPLOIDE}

Para explicar el modelo diploide, se partirá del modelo más simple que es dos alelos en un locus en dominancia completa. Donde $A$ se refiere al alelo dominante y $a$ se refiere al alelo recesivo. Supongamos que $p A$ es la proporción de gametos con el alelo $A$ y $q a$ es la proporción de gametos con el alelo $a$ y hay que tener en cuenta que $p+q=1$. Y que si se tiene una población panmíctica (sin selección, sin mutación, sin migración y en apareamiento aleatorio), se tendrá el equilibrio Hardy-Weinberg, donde:

$(p A+q a)^{2}=p^{2} A A+2 p q A a+q^{2} a a$ 1

Por lo que se tendrá una proporción de $p^{2}$ de homocigotos dominantes $(A A), 2 p q$ de heterocigotos $(A a)$ y de $q 2$ para los homocigotos recesivos $(a a)$, es decir, el cuadrado de la suma de la frecuencia de 
los gametos es igual a las frecuencias genotípicas, siguiendo esta lógica los gametos tienen uno y sólo un alelo (1). Sin embargo, cuando se tienen poliploides los gametos se complican ya que en un modelo con dos alelos en un locus octoploide se tendrían gametos tetraploides (cuatro copias del alelo $A / a$ ), por lo que en lugar de dos gametos estos serían cinco y se tendrían $p A A A A, q A A A a, r A A a a$, sAaaa y taaaa, donde $p+q+r+s+t=1$; y al aplicar la ley del equilibrio quedaría lo siguiente:

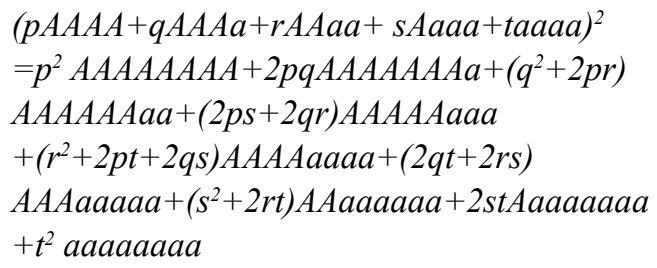

Donde, sí existe dominancia completa, quiere decir que con que uno de los ocho alelos del individuo fuera dominante entonces se expresaría dicha característica dominante, por lo que, a pesar de producir nueve genotipos diferentes, esto reduce a un solo genotipo con la característica recesiva, razón por la cual se presume la progenie sea muy parecida a los progenitores y se pueda confundir con individuos apomícticos.

No obstante, en un modelo diploide la frecuencia alélica es igual a frecuencia gamética, pero para el caso de que la frecuencia alélica fuera de $p A$ y qa para los alelos dominante y recesivo, respectivamente, entonces en un modelo octoploide se tendrían las siguientes frecuencias gaméticas y genotípicas: de los dos alelos, en el caso especial de los octoploides cuatro alelos $A$ y cuatro alelos $a$.

Al comparar diferentes niveles de ploidía en su heterocigocidad y en la probabilidad de obtener un genotipo con la característica recesiva en un modelo de dos alelos en dominancia completa donde $p=q=1 / 2$, se tendrían los resultados indicados en el cuadro 1.

Estos resultados hacen que, bajo el supuesto de dominancia completa, la progenie segregante sea muy homogénea en un gen de herencia simple teniendo la probabilidad de $1 / 256$ de encontrar un individuo que presente la característica recesiva.

\begin{tabular}{|c|c|c|c|c|}
\hline $\begin{array}{c}\text { Nivel } \\
\text { DE } \\
\text { PLOIDÍ́ }\end{array}$ & $\begin{array}{l}\text { NÚMERo } \\
\text { DE } \\
\text { GAMETOS }\end{array}$ & $\begin{array}{c}\text { NÚMERo } \\
\text { DE } \\
\text { GENOTIPOS }\end{array}$ & $\begin{array}{l}\text { Hetero- } \\
\text { Cigocidad }\end{array}$ & $\begin{array}{c}\text { Probabi- } \\
\text { LIDAD DEL } \\
\text { HOMOCI- } \\
\text { GOTO } \\
\text { RECESIVO } \\
\end{array}$ \\
\hline $2 n=2 x$ & 2 & 3 & $1 / 2$ & $1 / 4$ \\
\hline $2 n=4 x$ & 3 & 5 & $7 / 8$ & $1 / 16$ \\
\hline $2 n=6 x$ & 4 & 7 & $31 / 32$ & $1 / 64$ \\
\hline $2 n=8 x$ & 5 & 9 & $127 / 128$ & $1 / 256$ \\
\hline
\end{tabular}

Por lo tanto en un plan para formar un programa de mejoramiento genético de una especie octoploide, como lo es el nopal, existe el supuesto de una alta probabilidad de que el germoplasma recolectado sea

$$
\begin{aligned}
\left(\frac{4 !}{4 !(4-4) !} p^{4}\right. & A A A A+\frac{4 !}{3 !(4-3) !} p^{3} q A A A a+\frac{4 !}{2 !(4-2) !} p^{2} q^{2} A A a a \\
& \left.+\frac{4 !}{1 !(4-1) !} p q^{3} \text { Aaaa }+\frac{4 !}{0 !(4-0) !} q^{4} a a a a\right)^{2} \\
& =\left(p^{4} A A A A+4 p^{3} q A A A a+6 p^{2} q^{2} A A a a+4 p q^{3} \text { Aaaa }+q^{4} a a a a\right)^{2} \\
& =p^{8} A A A A A A A A+8 p^{7} q A A A A A A A a+28 p^{6} q^{2} \text { AAAAAAaa } \\
& +56 p^{5} q^{3} \text { AAAAAaaa }+70 p^{4} q^{4} \text { AAAAaaaa }+56 p^{3} q^{5} \text { AAAaaaaa } \\
& +28 p^{2} q^{6} \text { AAaaaaaa }+8 p q^{7} \text { Aaaaaaaa }+q^{8} \text { aaaaaaaa }
\end{aligned}
$$

Al maximizar el número de heterocigotos, es decir cuando $p=q=1 / 2$, en un modelo diploide se muestra que se tiene una heterocigocidad máxima de $1 / 2$ mientras que en un modelo octoploide se tiene que la heterocigocidad, dado por todas sus variantes heterocigóticas, suma ${ }^{127}{ }_{128^{\prime}}$ es decir, el genotipo presenta los dos alelos, aunque sea en diferentes proporciones, $\mathrm{y}{ }^{35} / 128$ cuando tienen el mismo número de repeticiones heterocigoto, y de que generará una alta segregación genotípica al hibridarlo; no obstante, bajo el supuesto de dominancia completa, pensando que con un solo alelo dominante se expresaría la característica fenotípica dominante, se tendrá una población segregante muy homogénea que podría fácilmente confundirse con una propagación vegetativa por semilla, es decir apomixis. 


\section{MODELO DE UN LOCUS CON ALELOS MÚLTIPLES EN UN OCTAPLOIDE}

Cuando se presenta un modelo con alelos múltiples, una planta octoploide podría contener hasta ocho diferentes alelos en un mismo locus en un individuo, lo que generaría tener 70 gametos diferentes obtenidos a través de sus combinaciones $\left(\begin{array}{l}8 \\ 4\end{array}\right)=\frac{8 !}{(8-4) ! 4 !}=70$ lo que generaría 2485 genotipos diferentes al autofecundar este individuo, obtenido por los genotipos diferentes de acuerdo a su matriz dialélica y donde no habría homocigotos en dicha población segregante; no obstante, con ocho alelos en la población se tendrían 330 gametos diferentes $\left(\frac{(8+4-1) !}{(8-1) ! \cdot 4 !}=330\right)$ y se tendrían 6435 genotipos diferentes $\left(\frac{(8+8-1) !}{(8-1) ! \cdot 8 !}=6435\right)$.

Al generar modelos de alelos múltiples en poliploides se debe tener en cuenta que un mismo individuo puede presentar todas las formas alélicas, a diferencia de los materiales diploides donde, aunque se encuentre un gen con alelos múltiples, un individuo sólo tiene dos alelos y la presencia de alelos múltiples es en la población, como el caso de la sangre en humanos donde se tienen tres alelos $\left(I_{A}, I_{B}\right.$ e $\left.i\right)$ y donde los alelos $I_{A}$ e $I_{B}$ presentan dominancia completa sobre $i$ y entre ellos presentan codominancia lo que refleja cuatro fenotipos distintos: los de sangre tipo $A\left(I_{A} I_{A}\right.$ e $\left.I_{A} i\right)$, los del tipo $B\left(I_{B} I_{B}\right.$ e $\left.I_{B} i\right)$, los del tipo $O(i i)$ y los del tipo $A B\left(I_{A} I_{B}\right)$. Estos efectos de dominancia completa, codominancia, sobredominancia y dominancia parcial podrían estar presentes en un solo gen en un individuo poliploide, lo que complica aún más poder entender su sistema hereditario.

Y los cálculos se complican en el momento de que dos gametos pueden generar un genotipo que otros dos gametos diferentes también lo generan, por lo que los cálculos se deben hacer para el caso de alelos múltiples es muy similar a lo que se genera para diploides, donde para el caso de tres alelos $\left(A_{1}, A_{2}\right.$ y $\left.A_{3}\right)$ con frecuencias alélicas $p_{1^{\prime}} p_{2}$ y $p_{3}\left(p_{1}+p_{2}+p_{3}=1\right)$ genera la siguiente segregación en un individuo diploide:

$$
\begin{aligned}
\left(p_{1} A_{1}+p_{2} A_{2}+p_{3} A_{3}\right)^{2} & \\
& =p_{1}^{2} A_{1} A_{1}+p_{2}^{2} A_{2} A_{2}+p_{3}^{2} A_{3} A_{3}+2 p_{1} p_{2} A_{1} A_{2}+2 p_{1} p_{3} A_{1} A_{3}+2 p_{2} p_{3} A_{2} A_{3}
\end{aligned}
$$

Y que después podemos generalizar de la siguiente manera para cualquier número de alelos múltiples:

$$
\left(\sum_{i=1}^{n} p_{i} A_{i}\right)^{2}=\sum_{i=1}^{n} p_{i}^{2} A_{i} A_{i}+2 \sum_{\substack{i, j=1 \\ \forall i \neq j}}^{n} p_{i} p_{j} A_{i} A_{j}
$$

Para el caso de un modelo octoploide con tres alelos se generan 15 diferentes gametos y 45 genotipos como se muestra a continuación:

$$
\begin{aligned}
& \left(\frac{4 !}{4 ! 0 ! 0 !} p_{1}^{4} A_{1} A_{1} A_{1} A_{1}+\frac{4 !}{0 ! 4 ! 0 !} p_{2}^{4} A_{2} A_{2} A_{2} A_{2}+\frac{4 !}{0 ! 0 ! 4 !} p_{3}^{4} A_{3} A_{3} A_{3} A_{3}+\frac{4 !}{3 ! 1 ! 0 !} p_{1}^{3} p_{2} A_{1} A_{1} A_{1} A_{2}+\frac{4 !}{3 ! 0 ! 1 !} p_{1}^{3} p_{3} A_{1} A_{1} A_{1} A_{3}\right. \\
& +\frac{4 !}{0 ! 3 ! 1 !} p_{2}^{3} p_{3} A_{2} A_{2} A_{2} A_{3}+\frac{4 !}{1 ! 3 ! 0 !} p_{1} p_{2}^{3} A_{1} A_{2} A_{2} A_{2}+\frac{4 !}{1 ! 0 ! 3 !} p_{1}^{3} p_{3} A_{1} A_{1} A_{1} A_{3} \\
& +\frac{4 !}{0 ! 1 ! 3 !} p_{2} p_{3}^{3} A_{2} A_{3} A_{3} A_{3}+\frac{4 !}{2 ! 2 ! 0 !} p_{1}^{2} p_{2}^{2} A_{1} A_{1} A_{2} A_{2}+\frac{4 !}{2 ! 0 ! 2 !} p_{1}^{2} p_{3}^{2} A_{1} A_{1} A_{3} A_{3} \\
& +\frac{4 !}{0 ! 2 ! 2 !} p_{2}^{2} p_{3}^{2} A_{2} A_{2} A_{3} A_{3}+\frac{4 !}{2 ! 1 ! 1 !} p_{1}^{2} p_{2} p_{3} A_{1} A_{1} A_{2} A_{3}+\frac{4 !}{1 ! 2 ! 1 !} p_{1} p_{2}^{2} p_{3} A_{1} A_{2} A_{2} A_{3} \\
& \left.+\frac{4 !}{1 ! 1 ! 2 !} p_{1} p_{2} p_{3}^{2} A_{1} A_{2} A_{3} A_{3}\right)^{2} \\
& =\left(p_{1}^{4} A_{1} A_{1} A_{1} A_{1}+p_{2}^{4} A_{2} A_{2} A_{2} A_{2}+p_{3}^{4} A_{3} A_{3} A_{3} A_{3}+4 p_{1}^{3} p_{2} A_{1} A_{1} A_{1} A_{2}+4 p_{1}^{3} p_{3} A_{1} A_{1} A_{1} A_{3}\right. \\
& +4 p_{2}^{3} p_{3} A_{2} A_{2} A_{2} A_{3}+4 p_{1} p_{2}^{3} A_{1} A_{2} A_{2} A_{2}+4 p_{1} p_{3}^{3} A_{1} A_{3} A_{3} A_{3}+4 p_{2} p_{3}^{3} A_{2} A_{3} A_{3} A_{3}+6 p_{1}^{2} p_{2}^{2} A_{1} A_{1} A_{2} A_{2} \\
& +6 p_{1}^{2} p_{3}^{2} A_{1} A_{1} A_{3} A_{3}+6 p_{2}^{2} p_{3}^{2} A_{2} A_{2} A_{3} A_{3}+12 p_{1}^{2} p_{2} p_{3} A_{1} A_{1} A_{2} A_{3}+12 p_{1} p_{2}^{2} p_{3} A_{1} A_{2} A_{2} A_{3} \\
& \left.+12 p_{1} p_{2} p_{3}^{2} A_{1} A_{2} A_{3} A_{3}\right)^{2} \\
& =p_{1}^{8} A_{1} A_{1} A_{1} A_{1} A_{1} A_{1} A_{1} A_{1}+p_{2}^{8} A_{2} A_{2} A_{2} A_{2} A_{2} A_{2} A_{2} A_{2}+p_{3}^{8} A_{3} A_{3} A_{3} A_{3} A_{3} A_{3} A_{3} A_{3} \\
& +8 p_{1}^{7} p_{2} A_{1} A_{1} A_{1} A_{1} A_{1} A_{1} A_{1} A_{2}+8 p_{1}^{7} p_{3} A_{1} A_{1} A_{1} A_{1} A_{1} A_{1} A_{1} A_{3}+8 p_{1} p_{2}^{7} A_{1} A_{2} A_{2} A_{2} A_{2} A_{2} A_{2} A_{2} \\
& +8 p_{2}^{7} p_{3} A_{2} A_{2} A_{2} A_{2} A_{2} A_{2} A_{2} A_{3}+8 p_{1} p_{3}^{7} A_{1} A_{3} A_{3} A_{3} A_{3} A_{3} A_{3} A_{3}+8 p_{2} p_{3}^{7} A_{2} A_{3} A_{3} A_{3} A_{3} A_{3} A_{3} A_{3} \\
& +28 p_{1}^{6} p_{2}^{2} A_{1} A_{1} A_{1} A_{1} A_{1} A_{1} A_{2} A_{2}+28 p_{1}^{6} p_{3}^{2} A_{1} A_{1} A_{1} A_{1} A_{1} A_{1} A_{3} A_{3}+28 p_{1}^{2} p_{2}^{6} A_{1} A_{1} A_{2} A_{2} A_{2} A_{2} A_{2} A_{2} \\
& +28 p_{2}^{6} p_{3}^{2} A_{2} A_{2} A_{2} A_{2} A_{2} A_{2} A_{3} A_{3}+28 p_{1}^{2} p_{3}^{6} A_{1} A_{1} A_{3} A_{3} A_{3} A_{3} A_{3} A_{3}+28 p_{2}^{2} p_{3}^{6} A_{2} A_{2} A_{3} A_{3} A_{3} A_{3} A_{3} A_{3} \\
& +56 p_{1}^{6} p_{2} p_{3} A_{1} A_{1} A_{1} A_{1} A_{1} A_{1} A_{2} A_{3}+56 p_{1} p_{2}^{6} p_{3} A_{1} A_{2} A_{2} A_{2} A_{2} A_{2} A_{2} A_{3} \\
& +56 p_{1} p_{2} p_{3}^{6} A_{1} A_{2} A_{3} A_{3} A_{3} A_{3} A_{3} A_{3}+56 p_{1}^{5} p_{2}^{3} A_{1} A_{1} A_{1} A_{1} A_{1} A_{2} A_{2} A_{2}+56 p_{1}^{5} p_{3}^{3} A_{1} A_{1} A_{1} A_{1} A_{1} A_{3} A_{3} A_{3} \\
& +56 p_{1}^{3} p_{2}^{5} A_{1} A_{1} A_{1} A_{2} A_{2} A_{2} A_{2} A_{2}+56 p_{1}^{3} p_{3}^{5} A_{1} A_{1} A_{1} A_{3} A_{3} A_{3} A_{3} A_{3}+56 p_{2}^{5} p_{3}^{3} A_{2} A_{2} A_{2} A_{2} A_{2} A_{3} A_{3} A_{3} \\
& +56 p_{2}^{3} p_{3}^{5} A_{2} A_{2} A_{2} A_{3} A_{3} A_{3} A_{3} A_{3}+168 p_{1}^{5} p_{2}^{2} p_{3} A_{1} A_{1} A_{1} A_{1} A_{1} A_{2} A_{2} A_{3} \\
& +168 p_{1}^{5} p_{2} p_{3}^{2} A_{1} A_{1} A_{1} A_{1} A_{1} A_{2} A_{3} A_{3}+168 p_{1}^{2} p_{2}^{5} p_{3} A_{1} A_{1} A_{2} A_{2} A_{2} A_{2} A_{2} A_{3} \\
& +168 p_{1} p_{2}^{5} p_{3}^{2} A_{1} A_{2} A_{2} A_{2} A_{2} A_{2} A_{3} A_{3}+168 p_{1}^{2} p_{2} p_{3}^{5} A_{1} A_{1} A_{2} A_{3} A_{3} A_{3} A_{3} A_{3} \\
& +168 p_{1} p_{2}^{2} p_{3}^{5} A_{1} A_{2} A_{2} A_{3} A_{3} A_{3} A_{3} A_{3}+70 p_{1}^{4} p_{2}^{4} A_{1} A_{1} A_{1} A_{1} A_{2} A_{2} A_{2} A_{2}+70 p_{1}^{4} p_{3}^{4} A_{1} A_{1} A_{1} A_{1} A_{3} A_{3} A_{3} A_{3} \\
& +70 p_{2}^{4} p_{3}^{4} A_{2} A_{2} A_{2} A_{2} A_{3} A_{3} A_{3} A_{3}+280 p_{1}^{4} p_{2}^{3} p_{3} A_{1} A_{1} A_{1} A_{1} A_{2} A_{2} A_{2} A_{3} \\
& +280 p_{1}^{4} p_{2} p_{3}^{3} A_{1} A_{1} A_{1} A_{1} A_{2} A_{3} A_{3} A_{3}+280 p_{1}^{3} p_{2}^{4} p_{3} A_{1} A_{1} A_{1} A_{2} A_{2} A_{2} A_{2} A_{3} \\
& +280 p_{1}^{3} p_{2} p_{3}^{4} A_{1} A_{1} A_{1} A_{2} A_{3} A_{3} A_{3} A_{3}+280 p_{1} p_{2}^{4} p_{3}^{3} A_{1} A_{2} A_{2} A_{2} A_{2} A_{3} A_{3} A_{3} \\
& +280 p_{1} p_{2}^{3} p_{3}^{4} A_{1} A_{2} A_{2} A_{2} A_{3} A_{3} A_{3} A_{3}+420 p_{1}^{4} p_{2}^{2} p_{3}^{2} A_{1} A_{1} A_{1} A_{1} A_{2} A_{2} A_{3} A_{3} \\
& +420 p_{1}^{2} p_{2}^{4} p_{3}^{2} A_{1} A_{1} A_{2} A_{2} A_{2} A_{2} A_{3} A_{3}+420 p_{1}^{2} p_{2}^{2} p_{3}^{4} A_{1} A_{1} A_{2} A_{2} A_{3} A_{3} A_{3} A_{3} \\
& +560 p_{1}^{3} p_{2}^{3} p_{3}^{2} A_{1} A_{1} A_{1} A_{2} A_{2} A_{2} A_{3} A_{3}+560 p_{1}^{3} p_{2}^{2} p_{3}^{3} A_{1} A_{1} A_{1} A_{2} A_{2} A_{3} A_{3} A_{3} \\
& +560 p_{1}^{2} p_{2}^{3} p_{3}^{3} A_{1} A_{1} A_{2} A_{2} A_{2} A_{3} A_{3} A_{3}
\end{aligned}
$$


Con esto se muestra que obtener una fórmula general para el caso de poliploides es realmente complejo. Para el caso de tres alelos suponiendo que uno sea recesivo, como el caso del alelo $i$ en la sangre en humanos, existe una probabilidad de $1 / 6561$ de obtener un homocigoto con este alelo recesivo y la probabilidad de tener un genotipo heterocigoto es de ${ }^{2186} / 2187$ en todas sus variantes. Por lo tanto, se necesitan poblaciones segregantes grandes para poder obtener variantes fenotípicas, de otra forma, probabilísticamente se genera la posibilidad de no encontrar estos fenotipos. No obstante, se propone la siguiente generalización para un octoploide con $n$ alelos:

$$
\begin{aligned}
& \left(\sum_{i=1}^{n} p_{i} A_{i}\right)^{8}=\sum_{i=1}^{n} p_{i}^{\mathrm{g}} A_{i} A_{i} A_{i} A_{i} A_{i} A_{i} A_{i} A_{l}+8 \sum_{\substack{j=1 \\
\forall \neq 1}}^{n} p_{i}^{7} p_{j} A_{i} A_{i} A_{i} A_{i} A_{i} A_{i} A_{i} A_{j}+28 \sum_{\substack{i, j=1 \\
\forall i \neq i}}^{n} p_{i}^{6} p_{j}^{2} A_{i} A_{i} A_{i} A_{i} A_{i} A_{i} A_{j} A_{j} \\
& +56 \sum_{\substack{l, j, k=1 \\
Y i \neq i \neq k}}^{n} p_{i}^{6} p_{j} p_{k} A_{i} A_{i} A_{l} A_{i} A_{i} A_{i} A_{j} A_{k}+56 \sum_{i, j=1}^{n} p_{l}^{5} p_{j}^{3} A_{i} A_{i} A_{i} A_{l} A_{i} A_{j} A_{j} A_{j}
\end{aligned}
$$

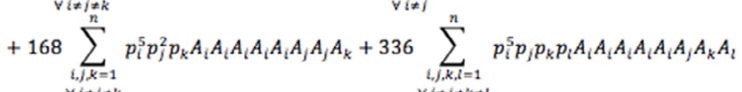

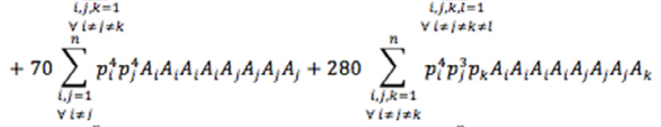

$$
\begin{aligned}
& +420 \sum_{\substack{i, j<=1 \\
\forall \neq i \neq k}}^{n} p_{i}^{4} p_{j}^{2} p_{k}^{2} A_{i} A_{i} A_{i} A_{l} A_{j} A_{j} A_{k} A_{k}+840 \sum_{\substack{i, j, l=1 \\
\forall \neq j \neq k \neq l}}^{n} p_{i}^{4} p_{j}^{2} p_{k} p_{l} A_{l} A_{i} A_{i} A_{l} A_{j} A_{j} A_{k} A_{l}
\end{aligned}
$$

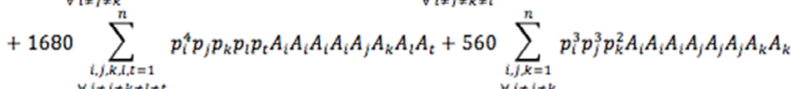

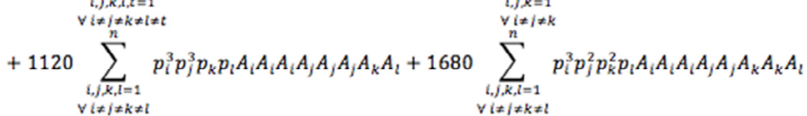

$$
\begin{aligned}
& +3360 \sum_{\substack{i, j, k, t, i=1 \\
\forall i \neq i \neq k \neq i \neq t}}^{\substack{v i \neq i \neq \neq l \\
n}} p_{i}^{3} p_{j}^{2} p_{k} p_{i} p_{t} A_{i} A_{i} A_{i} A_{j} A_{j} A_{k} A_{i} A_{t}
\end{aligned}
$$

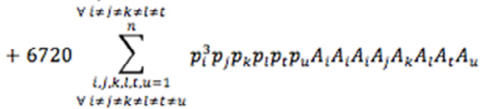

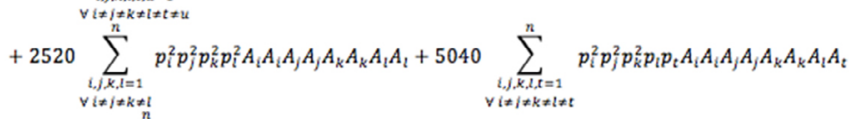

$$
\begin{aligned}
& +10080 \sum_{\substack{i, j, k, t, u=1 \\
\forall \neq i \neq k \neq \neq \neq \neq \neq}}^{n} p_{i}^{2} p_{j}^{2} p_{k} p_{t} p_{t} p_{u} A_{i} A_{i} A_{j} A_{j} A_{k} A_{l} A_{t} A_{u} \\
& +20160 \sum_{\substack{i, j, k, t, u, v=1 \\
\forall \neq i \neq k \neq i \neq t \neq u \neq v}}^{n} p_{i}^{2} p_{j} p_{k} p_{l} p_{t} p_{u} p_{v} A_{l} A_{i} A_{j} A_{k} A_{l} A_{l} A_{u} A_{v}
\end{aligned}
$$

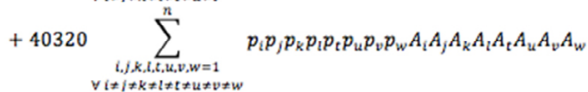

Por ejemplo, en un modelo octoploide de dos loci independientes con dos alelos cada uno, en dominancia completa, se tiene que la posibilidad de generar un individuo con ambos loci en homocigosis recesiva es de $1 / 65536$ el cual probablemente no encontraríamos si se tienen menos de 65,536 individuos segregantes y aun así existe la posibilidad de no encontrarlo por cuestiones de azar, por lo que necesitaríamos una población de alrededor de 70,000 individuos para poder tener certeza probabilística de encontrarlo.

Por lo que el número de alelos presentes en un locus va a generar diferentes probabilidades de heterocigotos y homocigotos, así como diferente número de gametos y genotipos a obtener sin importar el tipo de dominancia que se presente en cada caso. Aquí se calculó de 2 a 8 alelos por locus con la misma probabilidad de ocurrencia de cada alelo para mayor entendimiento, es decir, $p_{1}=p_{2}=p_{3}=\cdots=p_{n}$ (cuadro 2 ). 
Cuadro 2. Número de gametos y genotipos diferentes, así como probabilidad de homocigosis y heterocigosis en una población octoploide con todos los alelos con la misma frecuencia $p_{1}=p_{2}=p_{3}=\cdots=p_{n}$.

\begin{tabular}{ccccc}
\hline $\begin{array}{c}\text { NúMERO } \\
\text { DE } \\
\text { ALELOS }\end{array}$ & $\begin{array}{c}\text { NÚMERO } \\
\text { DE } \\
\text { GAMETOS }\end{array}$ & $\begin{array}{c}\text { NúMERO } \\
\text { DE } \\
\text { GENOTIPOS }\end{array}$ & $\begin{array}{c}\text { PROBABI- } \\
\text { LIDAD DE } \\
\text { HOMOCI- } \\
\text { GOSIS }\end{array}$ & $\begin{array}{c}\text { PROBABI- } \\
\text { LIDAD DE } \\
\text { HETERO- } \\
\text { CIGOSIS }\end{array}$ \\
\hline 2 & 5 & 9 & $1 / 128$ & $127 / 128$ \\
3 & 15 & 45 & $1 / 2187$ & $2186 / 2187$ \\
4 & 35 & 165 & $1 / 16384$ & $16383 / 16384$ \\
5 & 70 & 495 & $1 / 78125$ & $78124 / 78125$ \\
6 & 126 & 1287 & $1 / 279936$ & $279935 / 279936$ \\
7 & 210 & 3003 & $1 / 823543$ & $823542 / 823543$ \\
8 & 330 & 6435 & $1 / 2097152$ & $2097151 / 2097152$ \\
\hline
\end{tabular}

Como se observa la probabilidad de heterocigosis en un modelo octoploide es superior al 99\% sin importar el número de alelos, lo que genera poblaciones con muy alta heterocigocidad y el no conocer su valor genotípico, es decir, conocer si existe dominancia completa, codominancia, dominancia parcial o sobredominancia impide generar cruzas específicas para mejorar caracteres de herencia simple y complica saber cuánta de la variabilidad genética es por variación de dominancia y cuanta es por variación aditiva. Sin contar aún con los efectos ambientales e interacciones genotipo-ambiente específicas sino sólo y exclusivamente a la variación genética debida a la segregación de la herencia mendeliana.

\section{Discusión}

La mayoría de cultivares registrados de nopal son materiales muy homogéneos y se presume que esto se debe a que son propagados principalmente por medios vegetativos (Gallegos-Vázquez y Mondragón-Jacobo 2011); sin embargo, puede tratarse de muchos genotipos que generan el mismo fenotipo, lo cual en mejoramiento afecta de sobremanera debido a que si una cruza no se hace exactamente con el mismo individuo se pueden tener poblaciones segregantes diferentes aunque estén catalogadas como el mismo cultivar y se presuma que es el mismo genotipo. Asimismo, por lo desarrollado anteriormente se puede suponer que la mayoría de cultivares, si no es que todos, son mayormente heterocigotos por lo que al propagarlos sexualmente van a generar poblaciones segregantes de alta variabilidad, donde en esta segregación se pueden encontrar materiales distintos a los padres; no obstante el no conocer si algunas características de herencia simple presentan dominancia completa, codominancia, dominancia parcial o sobredominancia hacen que desarrollar un programa de mejoramiento genético por hibridación exitoso, como para el caso de muchas especies diploides, sea casi una misión imposible; más aún demostrar que alguna característica presenta herencia simple es complejo por el carácter poliploide del nopal y los cálculos que para ello se generan, debido a que se pueden probar diversas hipótesis de segregación mendeliana (diversos tipos de herencia y de número de alelos) y varias de ellas se pueden concluir como ciertas. Es por esto que se necesitan generar una mayor cantidad de estudios en nopal para empezar a descifrar los tipos de herencia que tienen diversos caracteres y su posible adaptación a hipótesis de herencia mendeliana.

Por otro lado, al hacer cruzas en distintos genotipos que están catalogadas dentro de la misma especie taxonómica como lo es el caso de 'Reyna' y 'Cristalina' (ambas catalogadas como O. albicarpa Scheinvar) se ha encontrado en la población segregante una alta homogeneidad, donde todos ellos fueron parecidos a la madre lo que fácilmente puede confundirse con procesos de propagación vegetativa como apomixis. Sin embargo, con el uso de marcadores ISSR a las plantas progenitoras y su progenie, Pacheco-Aguilar et al. (2012) mostraron que existen mayores índices de similaridad con el padre que con la madre, casos similares presentaron otras cruzas entre diversos cultivares de nopal (cuadro 3), donde todas ellas tuvieron similaridad con ambos padres, lo que se traduce en que todos ellos fueron producto de propagación sexual.

Mondragón-Jacobo y Bordelon (2002) mencionan que la presencia de apomixis puede corroborarse porque muchos individuos de la progenie presentaron perfiles idénticos, o casi idénticos; sin embargo, en el desarrollo inicial de un locus con dos alelos en un modelo octoploide se corroboró que hasta $35 / 128$ podrían presentar un genotipo idéntico y quedan dentro de los parámetros normales de una segregación mendeliana para un octoploide. Estos resultados y la falta de pruebas citogenéticas de la presencia de apomixis en Opuntia genera la posibilidad de suponer que la segregación obtenida en las diferentes cruzas hechas en este género se deba a una segregación mendeliana típica de un octoploide. Aunado a que la poca probabilidad de éxito al hibridar nopal se deba 
Cuadro 3. Índice de similaridad de Nei y Li (1979) entre veinte individuos segregantes y sus progenitores en cruzas de cultivares de Nopal (Opuntia spp.) usando tres iniciadores ISSR.

\begin{tabular}{|c|c|c|c|c|c|c|c|c|c|c|c|c|c|c|c|c|c|c|c|c|}
\hline \multicolumn{21}{|c|}{ Progenie } \\
\hline & 1 & 2 & 3 & 4 & 5 & 6 & 7 & 8 & 9 & 10 & 11 & 12 & 13 & 14 & 15 & 16 & 17 & 18 & 19 & 20 \\
\hline $\mathrm{RV}+$ & 0.49 & 0.45 & 0.58 & 0.43 & 0.49 & 0.36 & 0.42 & 0.36 & 0.42 & 0.34 & 0.42 & 0.45 & 0.34 & 0.43 & 0.43 & 0.40 & 0.47 & 0.38 & 0.47 & 0.43 \\
\hline $\mathrm{SM} \partial$ & 0.51 & 0.55 & 0.42 & 0.57 & 0.51 & 0.64 & 0.58 & 0.64 & 0.58 & 0.66 & 0.58 & 0.55 & 0.66 & 0.57 & 0.57 & 0.60 & 0.53 & 0.62 & 0.53 & 0.57 \\
\hline Ry $q$ & 0.49 & 0.44 & 0.46 & 0.46 & 0.46 & 0.32 & 0.32 & 0.54 & 0.49 & 0.44 & 0.61 & 0.29 & 0.51 & 0.51 & 0.49 & 0.37 & 0.49 & 0.49 & 0.46 & 0.54 \\
\hline $\mathrm{Cr} \delta$ & 0.51 & 0.56 & 0.54 & 0.54 & 0.54 & 0.68 & 0.68 & 0.46 & 0.51 & 0.56 & 0.39 & 0.71 & 0.49 & 0.49 & 0.51 & 0.63 & 0.51 & 0.51 & 0.54 & 0.46 \\
\hline $\mathrm{RV}$ 우 & 0.59 & 0.57 & 0.59 & 0.63 & 0.51 & 0.51 & 0.53 & 0.55 & 0.59 & 0.51 & 0.55 & 0.51 & 0.59 & 0.61 & 0.61 & 0.45 & 0.51 & 0.51 & 0.51 & 0.57 \\
\hline Xo $\hat{\sigma}$ & 0.41 & 0.43 & 0.41 & 0.37 & 0.49 & 0.49 & 0.47 & 0.45 & 0.41 & 0.49 & 0.45 & 0.49 & 0.41 & 0.39 & 0.39 & 0.55 & 0.49 & 0.49 & 0.49 & 0.43 \\
\hline $\mathrm{RV}$ 우 & 0.49 & 0.51 & 0.39 & 0.43 & 0.43 & 0.55 & 0.49 & 0.59 & 0.51 & 0.51 & 0.43 & 0.51 & 0.59 & 0.51 & 0.57 & 0.51 & 0.53 & 0.61 & 0.73 & 0.47 \\
\hline $\mathrm{Cr} \delta^{\pi}$ & 0.51 & 0.49 & 0.61 & 0.57 & 0.57 & 0.45 & 0.51 & 0.41 & 0.49 & 0.49 & 0.57 & 0.49 & 0.41 & 0.49 & 0.43 & 0.49 & 0.47 & 0.39 & 0.27 & 0.53 \\
\hline
\end{tabular}

$\mathrm{RV}=$ 'rojo vigor'; $\mathrm{SM}=$ 'roja San Martín'; $\mathrm{Ry}={ }^{\prime}$ reyna'; $\mathrm{Cr}={ }^{\prime}$ cristalina'; $\mathrm{Xo}_{\mathrm{o}}{ }^{\prime}$ xoconostle cuaresmeño'

precisamente a esta segregación y al desconocimiento sobre cuánto de la variación genética se deba a la variación genética aditiva y cuanto a la variación genética de dominancia. Además, para probar genes de herencia simple en nopal es más complicado por la cantidad de cálculos que se necesitan, por ejemplo, en este trabajo se generó un modelo con dos y tres alelos por locus, pero falta desarrollar modelos con más alelos y el estudio de la interacción de caracteres donde se presume que efectos pleitrópicos, epistáticos y de ligamiento puedan estar también presentes dentro de este género. No obstante, la apomixis aposporea (poliembrionía) y diplosporia (partenogénesis) pueden estar presentes en el nopal, sin embargo, la principal fuente de variación que impide una efectividad en un programa de mejoramiento genético se debe a la segregación mendeliana poliploide debido a las probabilidades aquí descritas, lo que dificulta encontrar el arquetipo de nopal por medio de hibridación.

\section{Conclusiones}

El nopal por el hecho de ser poliploide presenta alta heterocigocidad y genera para cada locus desde 9 genotipos diferentes (dos alelos) hasta 6435 genotipos diferentes (ocho alelos).
Al no conocer si existe en características de nopal dominancia completa, codominancia, dominancia parcial o sobredominancia es difícil predecir su herencia, como es posible en diploides, así como los cálculos para validar genes de herencia simple.

La apomixis aposporea (poliembrionía) y diplosporia (partenogénesis) pueden estar presentes en el nopal, sin embargo, la principal fuente de variación que impide una efectividad en un programa de mejoramiento genético se debe a la segregación mendeliana poliploide debido a las probabilidades aquí descritas, lo que dificulta encontrar el arquetipo de nopal por medio de hibridación.

\section{Agradecimientos}

Ala Universidad deGuanajuato por el apoyo financiero mediante el proyecto institucional para fortalecer la excelencia académica 012/2015. Asimismo, se agradece al PRODEP por el financiamiento mediante el proyecto DSA/103.5/15/7007 “Relaciones genéticas entre variedades comerciales de nopal mediante PCR-RFLP". 


\section{LITERATURA CITADA}

Gallegos-Vázquez C, Mondragón-Jacobo C. 2011. Cultivares selectos de tuna, de México al mundo. Chapingo: Servicio Nacional de Inspección y Certificación de Semillas - Secretaría de Agricultura, Ganadería, Desarrollo Rural, Pesca y Alimentación (SAGARPA) - Universidad Autónoma Chapingo (UACH).

Gallegos-Vázquez C, Barrientos-Priego AF, Reyes-Agüero JA, Núñez-Colín CA, Mondragón-Jacobo C. 2011. Clusters of commercial varieties of cactus pear and xoconostle using UPOV morphological traits. Journal of Professional Association for Cactus Development 13: 10-22.

Gallegos-Vázquez C, Reyes-Agüero JA, Mondragón-Jacobo C. 2009. An update on the evolution of the cactus pear industry in Mexico. Acta Horticulturae 811: 69-76. https://doi.org/10.17660/ActaHortic.2009.811.5

García-Aguilar M, Pimienta-Barrios E. 1996. Cytological evidences of agamospermy in Opuntia (Cactaceae). Heseltonia 4: 39-42.

Mondragón-Jacobo C. 2001a. Cactus pear breeding and domestication. Plant Breeding Reviews 20: 135-166. https://doi.org/10.1002/9780470650189.ch5

Mondragón-Jacobo C. 2001b. Verification of the apomictic origin of cactus pear (Opuntia spp., Cactaceae) seedlings of open pollinated and crosses from Central Mexico. Journal of the Professional Association for Cactus Development 4: 49-56.

Mondragón-Jacobo C, Bordelon B. 2002. Presencia de apomixis en cruzas de nopales mexicanos y su identificación molecular preliminar. Revista Fitotecnia Mexicana 25(3): 247-252.
Nei M, Li WH. 1979. Mathematical model for studying genetic variation in terms of restriction endonucleases. Proceedings of the National Academy of Science (USA) 76: 5269-5273.

Pacheco-Aguilar MA, Mondragón-Jacobo C, Núñez-Colín CA, Villordo-Pineda E. 2012. Discriminación de plantas de origen sexual de nopal (Opuntia spp.) obtenidas de hibridaciones de variedades mexicanas mediante marcadores ISSR. En: J. A. Cueto Wong, R. G. Ávalos-Martínez \& D. Hernández Martínez (Comp.) Memoria de la VII Reunión Nacional de Innovación Agrícola (p. 208). Querétaro: Instituto Nacional de Investigaciones Forestales Agrícolas y Pecuarias.

Segura S, Scheinvar L, Olalde G, Leblanc O, Filardo S, Muratalla A, Gallegos C, Flores C. 2007. Genome sizes and ploidy levels in Mexican cactus pear species Opuntia (Tourn.) Mill. series Streptacanthae Britton et Rose, Leucotrichae DC., Heliabravoanae Scheinvar and Robustae Britton et Rose. Genetic Resources and Crop Evolution 54(5): 1033-1041. https://doi.org/10.1007/ s10722-006-9196-z.

Velez-Gutiérrez C, Rodríguez-Garay B. 1996. Microscopic Analysis of Polyembryony in Opuntia ficus-indica. Journal of the Professional Association for Cactus Development 1: 39-48. 